\title{
Uniform estimates with data from generalized Lebesgue spaces in periodic structures
}

\section{Yunsoo Jang ${ }^{1 *}$ (i)}

\section{"Correspondence:}

yunsoojang@kangwon.ac.kr 'Department of Mathematics Education, Kangwon National University, Chuncheon 24341, Republic of Korea

\begin{abstract}
We study various types of uniform Calderón-Zygmund estimates for weak solutions to elliptic equations in periodic homogenization. A global regularity is obtained with respect to the nonhomogeneous term from weighted Lebesgue spaces, Orlicz spaces, and weighted Orlicz spaces, which are generalized Lebesgue spaces, provided that the coefficients have small BMO seminorms and the domains are $\delta$-Reifenberg domains.
\end{abstract}

MSC: 46E30; 35B27; 35J15; 35D30

Keywords: Homogenization; Uniform estimate; Weighted Lebesgue space; Orlicz space; Reifenberg domain; BMO coefficient

\section{Introduction}

In this study, our problem is homogenization of elliptic equations of the form

$$
\begin{cases}D_{i}\left(A_{i j}^{\epsilon}(x) D_{j} u_{\epsilon}(x)\right)=D_{i} f_{i}(x) & \text { in } \Omega, \\ u_{\epsilon}(x)=0 & \text { on } \partial \Omega\end{cases}
$$

for $1 \leq i, j \leq n$ with $n \geq 2$, and $0<\epsilon \leq 1$. The given nonhomogeneous term is a vectorvalued function $f=\left\{f_{i}\right\}$ and the unknown is $u_{\epsilon}$. $\Omega$ is a bounded open set in $\mathbb{R}^{n}$ and the matrix of the coefficients is $A=\left\{A_{i j}\right\}, A_{i j}: \mathbb{R}^{n} \rightarrow \mathbb{R}$. To consider homogenization, we define $A^{\epsilon}=\left\{A_{i j}^{\epsilon}\right\}$ for each $0<\epsilon \leq 1$ by

$$
A_{i j}(x)=A_{i j}^{1}(x) \quad \text { and } \quad A_{i j}^{\epsilon}(x)=A_{i j}\left(\frac{x}{\epsilon}\right) \quad\left(x \in \mathbb{R}^{n}\right) .
$$

As usual, we assume that the coefficients are uniformly elliptic and uniformly bounded, that is, there exist positive constants $\lambda$ and $\Lambda$ such that

$$
\lambda|\xi|^{2} \leq A_{i j}(x) \xi_{i} \xi_{j} \quad \text { and } \quad\|A\|_{L^{\infty}} \leq \Lambda
$$

(c) The Author(s) 2021. This article is licensed under a Creative Commons Attribution 4.0 International License, which permits use, sharing, adaptation, distribution and reproduction in any medium or format, as long as you give appropriate credit to the original author(s) and the source, provide a link to the Creative Commons licence, and indicate if changes were made. The images or other third party material in this article are included in the article's Creative Commons licence, unless indicated otherwise in a credit line to the material. If material is not included in the article's Creative Commons licence and your intended use is not permitted by statutory regulation or exceeds the permitted use, you will need to obtain permission directly from the copyright holder. To view a copy of this licence, visit http://creativecommons.org/licenses/by/4.0/. 
for every $\xi \in \mathbb{R}^{n}$ and almost every $x \in \mathbb{R}^{n}$. Additionally, we consider periodic homogenization with the periodicity condition on $A$ :

$$
A(x+z)=A(x) \quad\left(x \in \mathbb{R}^{n}, z \in \mathbb{Z}^{n}\right) .
$$

Under these settings, our goal is to show uniform Calderón-Zygmund estimates for the weak solutions $u_{\epsilon}$ with respect to the given nonhomogeneous term $f$ from various spaces, weighted Lebesgue spaces, Orlicz spaces, and weighted Orlicz spaces. Here, uniform estimates mean that the estimates derived from our argument are independent of $\epsilon, 0<\epsilon \leq 1$. In addition, we study (1.1) with rapidly oscillating coefficients with discontinuity in nonsmooth domains. In fact, even though our method in this research is applicable to elliptic systems, see [4], and conormal derivative problems, which are a generalization of Neumann problems in nonsmooth domains, see [6], we here consider elliptic equations for simplicity in order to focus on various types of Calderón-Zygmund estimates.

Before starting our argument, we introduce some notations and definitions that are used throughout this paper.

\section{Notations 1.1}

(1) The open ball in $\mathbb{R}^{n}$ with center $y$ and radius $\rho>0$ is denoted by

$$
B_{\rho}(y)=\left\{x \in \mathbb{R}^{n}:|x-y|<\rho\right\} .
$$

If the center is the origin, we denote $B_{\rho}(0)$ by $B_{\rho}$.

(2) The integral average of $g \in L^{1}(U)$ over the bounded domain $U$ in $\mathbb{R}^{n}$ is denoted by

$$
(g)_{U}=\int_{U} g(x) d x=\frac{1}{|U|} \int_{U} g(x) d x
$$

(3) $B_{\rho}^{+}=B_{\rho} \cap\left\{x_{n}>0\right\}, B_{\rho}^{+}(y)=B_{\rho}^{+}+y, T_{\rho}=B_{\rho} \cap\left\{x_{n}=0\right\}, T_{\rho}(y)=T_{\rho}+y$.

(4) $\Omega_{\rho}(y)=B_{\rho}(y) \cap \Omega, \Omega_{\rho}=\Omega_{\rho}(0), \partial_{w} \Omega_{\rho}(y)=B_{\rho}(y) \cap \partial \Omega, \partial_{w} \Omega_{\rho}=\partial_{w} \Omega_{\rho}(0)$.

(5) For a set $E \subset \mathbb{R}^{n},|E|$ denotes the Lebesgue measure of $E$.

(6) $W^{1, p}(U)=\left\{g(x): D^{v} g \in L^{p}(U)\right.$ for each multi-index $v$ with $\left.|v| \leq 1\right\}$ is the Sobolev spaces equipped with the norm $\|g\|_{W^{1, p}(U)}=\|g\|_{L^{p}(U)}+\|D g\|_{L^{p}(U)}$. When $p=2$, we denote $H^{1}(U)=W^{1, p}(U)$. The closure of the space $C_{0}^{\infty}(U)$ with respect to the norm in $W^{1, p}(U)$ is denoted by $W_{0}^{1, p}(U)$.

The following is the definition of weak solutions to (1.1).

Definition 1.2 Let $f \in L^{2}(\Omega)$. Then $u_{\epsilon} \in H_{0}^{1}(\Omega)$ is called a weak solution to (1.1) if

$$
\int_{\Omega} A_{i j}^{\epsilon} D_{i} u_{\epsilon} D_{j} \phi d x=\int_{\Omega} f_{i} D_{i} \phi d x
$$

holds for all $\phi \in H_{0}^{1}(\Omega)$.

The next definitions cover the regularity requirements on the coefficient $A$, which allows discontinuity, and on the boundary of the domain $\Omega$, which is a natural extension of Lipschitz domains with small Lipschitz constants, see [33]. 
Definition 1.3 We say that $A$ is $(\delta, R)$-vanishing if

$$
\sup _{0<\rho \leq R} \sup _{y \in \mathbb{R}^{n}} f_{B_{\rho}(y)}\left|A(x)-(A)_{B_{\rho}(y)}\right|^{2} d x \leq \delta^{2} .
$$

Definition 1.4 We say that $\Omega$ is $(\delta, R)$-Reifenberg flat if, for every $x \in \partial \Omega$ and every $\rho \in$ $(0, R]$, there exists a coordinate system $\left\{y_{1}, \ldots, y_{n}\right\}$ depending on $\rho$ and $x$ so that $x=0$ in this coordinate system and

$$
B_{\rho} \cap\left\{y_{n}>\delta \rho\right\} \subset B_{\rho} \cap \Omega \subset B_{\rho} \cap\left\{y_{n}>-\delta \rho\right\} .
$$

Remark 1.5 First of all, the number $R$ in the above two definitions can be 1 or any other constant greater than 1 to be determined by our purpose, since our problems (1.1), (1.3), and (1.4) have a scaling invariance property. Also, the small constant $\delta$ to be determined later is scaling invariant. For (1.4), Reifenberg flatness is meaningful when $0<\delta<\frac{1}{2^{n+1}}$ for $n \geq 2$, see [33]. Even though this condition does not mean any smoothness condition on the boundary, this implies the following measure density condition:

$$
\frac{\left|B_{r}(y)\right|}{\left|B_{r}(y) \cap \Omega\right|} \leq\left(\frac{2}{1-\delta}\right)^{n} \leq\left(\frac{16}{7}\right)^{n}
$$

for every $y \in \partial \Omega$ and $r \in(0, R]$.

To understand our result in this work intuitively, we introduce a two-scale expansion method in homogenization, see [2]. For this, we split the variable $x$ into a macroscopic variable $x$ and a microscopic variable $y=\frac{x}{\epsilon}$. Then, according to the two-scale expansion $u_{\epsilon}(x)=u_{\epsilon}\left(x, \frac{x}{\epsilon}\right)=u_{\epsilon}(x, y)$, we see that

$$
\begin{aligned}
u_{\epsilon}(x, y) & =u_{0}(x, y)+\epsilon u_{1}(x, y)+\epsilon^{2} u_{2}(x, y)+\cdots \\
& =u_{0}(x)+\epsilon \chi_{i}(y) D_{i} u_{0}(x)+O\left(\epsilon^{2}\right) .
\end{aligned}
$$

Here, $u_{0}$ is independent of $y$ and $\chi=\left\{\chi_{i}(y)\right\}$ is called a corrector of (1.1) which is periodic. In fact, since only with (1.2) the corrector $\chi$ has nice estimates such as

$$
\|\chi\|_{L^{\infty}\left([0,1]^{n}\right)} \leq c \quad \text { or } \quad\|\chi\|_{H^{1}\left([0,1]^{n}\right)} \leq c
$$

we can think that

$$
u_{\epsilon}(x) \approx u_{0}(x)
$$

In addition, $u_{0}$ is a solution to

$$
\begin{cases}D_{i}\left(A_{i j}^{0} D_{j} u_{0}(x)\right)=D_{i} f_{i}(x) & \text { in } \Omega, \\ u_{0}(x)=0 & \text { on } \partial \Omega\end{cases}
$$

whose coefficient $A^{0}=\left\{A_{i j}^{0}\right\}$ is a constant matrix, and hence we may expect that integrability of $D u_{\epsilon}$ could be improved to the integrability of $f$ independent of $\epsilon$. However, this 
is in a heuristic way but not mathematical, so we need mathematical justification. In fact, $u_{0}$ is only the weak limit of the weak solutions $u_{\epsilon}$ in $H_{0}^{1}(\Omega)$ as $\epsilon \rightarrow 0$.

In the previous research [4], we proved that under suitable assumptions related to (1.3) and (1.4)

$$
f \in L^{p}(\Omega) \quad \Rightarrow \quad D u_{\epsilon} \in L^{p}(\Omega) \quad \text { with the estimate }\left\|D u_{\epsilon}\right\|_{L^{p}(\Omega)} \leq c\|f\|_{L^{p}(\Omega)}
$$

for $2<p<\infty$, where the constant $c$ is independent of $\epsilon$. In a bounded domain $f \in L^{p}(\Omega)$ for $2<p<\infty$ implies $f \in L^{2}(\Omega)$, so the Lax-Milgram lemma shows existence and uniqueness of the solutions $u_{\epsilon} \in H_{0}^{1}(\Omega)$ with the estimate

$$
\left\|D u_{\epsilon}\right\|_{L^{2}(\Omega)} \leq c\|f\|_{L^{2}(\Omega)}
$$

for some constant $c$ independent of $\epsilon$. Then, by using perturbation method, we can improve integrability of $D u_{\epsilon}$ from $L^{2}(\Omega)$ to $L^{p}(\Omega)$. In this sense, our goal is to prove that if $f \in X(\Omega) \subset L^{2}(\Omega)$, then

$$
f \in X(\Omega) \quad \Rightarrow \quad D u_{\epsilon} \in X(\Omega) \quad \text { with the estimate }\left\|D u_{\epsilon}\right\|_{X(\Omega)} \leq c\|f\|_{X(\Omega)}
$$

for some constant $c$ independent of $\epsilon$. In particular, $X(\Omega)$ will be weighted Lebesgue spaces, Orlicz spaces, or weighted Orlicz spaces defined on $\Omega$ which are generalizations of the Lebesgue space $L^{p}(\Omega)$.

For a single equation, which means $\epsilon=1$, the Calderón-Zygmund theory for the data from generalized Lebesgue spaces is widely and extensively studied even for nonlinear equations, higher-order equations, etc. We refer to [3, 7-12, 15, 16, 19, 20, 25, 27, 30, 32, $34,35]$ and the references therein for related results. Based on these results, in this article we want to extend uniform $L^{p}$ regularity results studied in $[1,4,14,31]$ to generalized spaces. The main difference which arises in the proof is that to obtain uniform estimates in the perturbation method we cannot compare our equation (1.1) to the equation whose coefficient is given by a constant matrix, such as the average value of $A$, as the proofs in the single equation cases. For this reason, different from the single equation case, in the perturbation argument we have to compare (1.1) with (3.6) whose weak solution does not have uniform $W^{1, \infty}$ regularity. Instead, we can derive our desired regularity results from uniform $W^{1 . q}(2<q<\infty)$ estimates for weak solutions to (3.6), see Lemma 3.3. In addition, through this procedure we apply their own properties of generalized Lebesgue spaces more delicate than single equation cases.

This paper is organized as follows. In Sect. 2, we introduce some useful results in weighted Lebesgue spaces, and in Sect. 3 we prove uniform Calderón-Zygmund estimates in weighted Lebesgue spaces. In the way to prove, we introduce an explicit and quantitative study in the approximation procedure. Orlicz spaces are considered in Sect. 4. Finally, in Sect. 5, we obtain the estimate in weighted Orlicz spaces.

\section{Auxiliary results in weighted Lebesgue spaces}

To begin with, we introduce the Hardy-Littlewood maximal function which is used in our approach. The Hardy-Littlewood maximal function is given by

$$
(\mathcal{M} g)(x)=\sup _{\rho>0} \frac{1}{\left|B_{\rho}(x)\right|} \int_{B_{r}(x)}|g(y)| d y
$$


for any locally integrable function $g$ on $\mathbb{R}^{n}$. If $g$ is defined only on a bounded subset of $\mathbb{R}^{n}$, then we define

$$
\mathcal{M} g=\mathcal{M} \bar{g}
$$

where $\bar{g}$ is the zero extension of $g$ from the bounded set to $\mathbb{R}^{n}$, and we define the restricted maximal function

$$
\mathcal{M}_{U} g=\mathcal{M}\left(g \mathbb{1}_{U}\right)
$$

where $\mathbb{1}_{U}$ is the characteristic function of $U \subset \mathbb{R}^{n}$. The main properties of this maximal function used in our argument are weak 1-1 estimate and strong $p$ - $p$ estimate.

Now we introduce weighted Lebesgue spaces with respect to the measure $w d x$. Especially, we consider the weight $w$ is in the Muckenhoupt class $A_{s}$ with $1<s<\infty$, see [26].

Definition 2.1 We say that $w$ is a weight in $A_{s}$ (or an $A_{s}$ weight) for $1<s<\infty$ if $w$ is a nonnegative and locally integrable function in $\mathbb{R}^{n}$ satisfying

$$
[w]_{s}=\sup _{y \in \mathbb{R}^{n}} \sup _{\rho>0}\left(f_{B_{\rho}(y)} w(x) d x\right)\left(f_{B_{\rho}(y)} w(x)^{\frac{-1}{s-1}} d x\right)^{s-1}<\infty .
$$

A typical example of $w \in A_{s}$ is

$$
w(x)=|x|^{q}-n<q<n(s-1) .
$$

For this weight $w$, the weighted Lebesgue measure with respect to $w$ is defined by

$$
w(E)=\int_{E} w(x) d x
$$

for measurable sets $E \in \mathbb{R}^{n}$ and the weighted Lebesgue space $L_{w}^{s}(U)$ is defined by the set of all measurable functions $g$ on $U$ satisfying

$$
\int_{U}|g(x)|^{s} w(x) d x<\infty
$$

Note that $L_{w}^{s}(U)$ is a Banach space equipped with the norm

$$
\|g\|_{L_{w}^{s}(U)}=\left(\int_{U}|g(x)|^{s} w(x) d x\right)^{\frac{1}{s}} .
$$

The next lemma is a relation between integration and summation, which comes from the classical measure theory.

Lemma 2.2 ([13]) Assume that $g$ is a nonnegative, measurable function defined on a bounded domain $\Omega \subset \mathbb{R}^{n}$. Let $\theta>0$ and $\mu>1$ be constants. Then, for $0<q<\infty$, we have

$$
g \in L^{q}(\Omega) \quad \Longleftrightarrow \quad S=\sum_{k \geq 1} \mu^{q k}\left|\left\{x \in \Omega: g(x)>\theta \mu^{k}\right\}\right|<\infty
$$


and

$$
\frac{1}{c} S \leq\|g\|_{L^{q}(\Omega)}^{q} \leq c(|\Omega|+S)
$$

where the constant $c$ depends only on $\theta, \mu$, and $q$.

Lemma 2.2 for the weighted Lebesgue spaces is the following.

Lemma 2.3 ([25]) Assume that $g$ is a nonnegative, measurable function defined on a bounded domain $\Omega \subset \mathbb{R}^{n}$. Let $\theta>0$ and $\mu>1$ be constants. Then, for $0<q<\infty$, we have

$$
g \in L_{w}^{q}(\Omega) \quad \Longleftrightarrow \quad S=\sum_{k \geq 1} \mu^{q k} w\left(\left\{x \in \Omega: g(x)>\theta \mu^{k}\right\}\right)<\infty
$$

and

$$
\frac{1}{c} S \leq\|g\|_{L_{w}^{q}(\Omega)}^{q} \leq c(w(\Omega)+S)
$$

where the constant $c$ depends only on $\theta, \mu, q$, and $w$.

Recalling Definition 1.4, we now introduce the Vitali type covering lemma for weighted Lebesgue spaces with weight $w \in A_{s}$.

Lemma 2.4 ([9]) Let $w \in A_{s}$ for some $1<s<\infty$. Assume that $\Omega$ is $(\delta, 1)$-Reifenberg flat, and let $\mathcal{C}$ and $\mathcal{D}$ be measurable sets with $\mathcal{C} \subset \mathcal{D} \subset \Omega$. We further assume that there exists small $\eta>0$ with

1. for each $x \in \Omega$,

$$
w\left(B_{1}(x) \cap \mathcal{C}\right)<\eta w\left(B_{1}(x)\right)
$$

2. for each $x \in \Omega$ and $\rho \in(0,1]$ with $w\left(\mathcal{C} \cap B_{\rho}(x)\right)>\eta w\left(B_{\rho}(x)\right)$, we have

$$
B_{\rho}(x) \cap \Omega \subset \mathcal{D} \text {. }
$$

Then

$$
w(\mathcal{C}) \leq c_{*} \eta w(\mathcal{D})
$$

for some constant $c_{*}=c_{*}(n, s, w)$.

Remark 2.5 In Lemma 2.4, we note that the constant $c_{*}$ depends on $\frac{1}{1-\delta}$. According to [33], $\delta$ is smaller than $\frac{1}{2^{n+1}}$. For this reason, we assume throughout this paper that $\delta<\frac{1}{2^{n+1}} \leq \frac{1}{8}$. In this sense, we can take the constant $c_{*}$ independent of $\delta$, which means that $c_{*}$ is a universal constant.

We will use some properties of the weighted Lebesgue measure with weight $w \in A_{s}$. The following lemma says that there is a relation between $w(x) d x$ and $d x$, the usual Lebesgue measure. 
Lemma 2.6 ([25]) Assume $w \in A_{s}$ for some $1<s<\infty$, and let $E$ be a measurable subset of a ball $B \subset \mathbb{R}^{n}$. Then, there exist positive constants $\alpha$ and $\tau \in(0,1)$, both depending on $n, s$, and $w$, such that

$$
\frac{1}{[w]_{s}}\left(\frac{|E|}{|B|}\right)^{s} \leq \frac{w(E)}{w(B)} \leq \alpha\left(\frac{|E|}{|B|}\right)^{\tau} .
$$

We note that, for the case $w=1$, the second part of (2.2) holds for any $\alpha>1$ and $0<\tau<1$ but it is natural to consider this inequality as an equality with $\alpha=1$ and $\tau=1$. Also, there is a fundamental property of the Hardy-Littlewood maximal function in the weighted Lebesgue spaces.

Lemma 2.7 ([26]) Assume $w \in A_{s}$ for some $1<s<\infty$. Then there is a constant $c=$ $c\left(n, s,[w]_{s}\right)>0$ such that

$$
c^{-1}\|g\|_{L_{w}^{s}\left(\mathbb{R}^{n}\right)} \leq\|\mathcal{M} g\|_{L_{w}^{s}\left(\mathbb{R}^{n}\right)} \leq c\|g\|_{L_{w}^{s}\left(\mathbb{R}^{n}\right)}
$$

for all $g \in L_{w}^{s}\left(\mathbb{R}^{n}\right)$.

\section{Uniform estimates in weighted Lebesgue spaces}

Our main theorem in this section is the following.

Theorem 3.1 Let $w \in A_{\frac{p}{2}}$ with $2<p<\infty$ and assume $f \in L_{w}^{p}(\Omega)$. Then there exists a small positive constant $\delta=\delta(\lambda, \Lambda, n, p, w)$ such that if $A=\left\{A_{i j}\right\}$ is $(\delta, 96)$-vanishing and $\Omega$ is $(\delta, 96)$ Reifenbergflat, then the weak solution $u_{\epsilon} \in H_{0}^{1}(\Omega)$ to (1.1) satisfies

$$
D u_{\epsilon} \in L_{w}^{p}(\Omega)
$$

with the estimate

$$
\left\|D u_{\epsilon}\right\|_{L_{w}^{p}(\Omega)} \leq c\|f\|_{L_{w}^{p}(\Omega)}
$$

for some constant $c=c(\operatorname{diam}(\Omega), \lambda, \Lambda, n, p, w, w(\Omega))$ which is independent of $\epsilon$.

Our method is based on localization and perturbation argument. To do this, we first consider the localized version of (1.1)

$$
\begin{cases}D_{i}\left(A_{i j}^{\epsilon}(x) D_{j} u_{\epsilon}(x)\right)=D_{i} f_{i}(x) & \text { in } \Omega_{6}, \\ u_{\epsilon}(x)=0 & \text { on } \partial_{w} \Omega_{6} .\end{cases}
$$

In (3.2), according to the Reifenberg flatness condition (1.4) we assume that

$$
B_{6}^{+} \subset \Omega_{6} \subset B_{6} \cap\left\{x_{n}>-12 \delta\right\}
$$

and a weak solution $u_{\epsilon}$ to (3.2) satisfies

$$
\frac{1}{\left|B_{6}\right|} \int_{\Omega_{6}}\left|D u_{\epsilon}\right|^{2} d x \leq 1
$$


We next let $w_{\epsilon}$ be a weak solution to

$$
\begin{cases}D_{i}\left(A_{i j}^{\epsilon}(x) D_{j} w_{\epsilon}(x)\right)=0 & \text { in } \Omega_{5}, \\ w_{\epsilon}(x)=u_{\epsilon}(x) & \text { on } \partial \Omega_{5} .\end{cases}
$$

Then, from (3.4), as we can see in the following lemma, we have a higher integrability result for $w_{\epsilon}$. In fact, since in the original version of the following lemma the authors considered very rough domains so that our domain, an intersection of a ball and a Reifenberg flat domain, which satisfies measure density condition (1.5) in Remark 1.5, is in the case of the original one, see [21]. More precisely, even though in (3.3) $\Omega_{6}$ itself may not be a Reifenberg flat domain, under the definition of Reifenberg domains in every scale, we have to approximate our domain in the same way as (3.3) and eventually it describes the boundary of Reifenberg domains from every scale with the measure density condition (1.5). In addition, under our $L^{2}$ approach in this paper, (1.5) implies the $p$-capacity condition with $p=2$, see [24, Sect. 2.2.3], we can apply [21] to our problem.

Lemma 3.2 ([21]) Assume (3.3) and let $u_{\epsilon}$ be a weak solution to (3.2) satisfying (3.4), and let $w_{\epsilon}$ be a weak solution to (3.5). Then there exist positive constants $\sigma_{1} \ll 1$ and c, which depend only on $\lambda, \Lambda$, and $n$ such that

$$
\left\|D w_{\epsilon}\right\|_{L^{2+\sigma_{1}\left(\Omega_{4}\right)}} \leq c
$$

Then we consider the following equation up to the flat boundaries:

$$
\begin{cases}D_{i}\left(A_{i j}^{\epsilon}(x) D_{j} v_{\epsilon}(x)\right)=0 & \text { in } B_{4}^{+}, \\ v_{\epsilon}(x)=0 & \text { on } T_{4} .\end{cases}
$$

According to the previous works $[4,5,18,31]$, we have the following lemma.

Lemma 3.3 Let $v_{\epsilon}$ be a weak solution to (3.6) and $2<q<\infty$. Then there exists $\delta=$ $\delta(\lambda, \Lambda, n, q)$ such that if $A$ is $(\delta, 6)$-vanishing, then we have

$$
D v_{\epsilon} \in L^{q}\left(B_{3}^{+}\right)
$$

with the estimate

$$
\left(f_{B_{3}^{+}}\left|D v_{\epsilon}\right|^{q} d x\right)^{\frac{1}{q}} \leq c\left(f_{B_{4}^{+}}\left|D v_{\epsilon}\right|^{2} d x\right)^{\frac{1}{2}}
$$

for some constant $c=c(\lambda, \Lambda, n, q)$ independent of $\epsilon$.

We here point out that estimate (3.7) is a uniform one for each given $2<q<\infty$. In fact, Lemma 3.3 is very important in our method since all the $\epsilon$ independent uniform estimates in this paper, (3.1), (4.4), and (5.1), come from this lemma whose proof can be found in [6, 31]. To complete our perturbation argument, we have to choose a weak solution $v_{\epsilon}$ to (3.6) suitably with respect to $w_{\epsilon}$, which is a weak solution to (3.5). For this, in the previous work 
[4] we used a compactness argument which is an indirect one, but we here find an explicit approximate solution $v_{\epsilon}$ to (3.6) with respect to $w_{\epsilon}$ based on the method in [22]. This is not only a direct method but also a quantitative study for the approximation procedure in Reifenberg domains.

Lemma 3.4 Assume (3.3). Let $u_{\epsilon}$ be a weak solution to (3.2) satisfying (3.4) and $w_{\epsilon}$ be the weak solution to (3.5). Then, for any fixed $\kappa>0$, there exists small $\delta=\delta(\kappa, \lambda, \Lambda, n)>0$ in (3.3) such that there exists a weak solution $v_{\epsilon}$ to (3.6) with

$$
\frac{1}{\left|B_{4}\right|} \int_{B_{4}^{+}}\left|D v_{\epsilon}\right|^{2} d x \leq c
$$

for some positive constant $c=c(\lambda, \Lambda, n)$ and

$$
\frac{1}{\left|B_{4}\right|} \int_{B_{4}^{+}}\left|D\left(w_{\epsilon}-v_{\epsilon}\right)\right|^{2} d x \leq \kappa^{2}
$$

Proof Firstly, we note that, according to (3.4),

$$
\frac{1}{\left|B_{5}\right|} \int_{\Omega_{5}}\left|D w_{\epsilon}\right|^{2} d x \leq c
$$

for some constant $c=c(\lambda, \Lambda, n)$ by the standard $L^{2}$ energy estimate for (3.5).

Next, we let $\phi$ be a smooth function that satisfies

$$
0 \leq \phi \leq 1, \quad \phi=1 \quad \text { on }\left\{x_{n} \geq 6 \delta\right\}, \quad \phi=0 \quad \text { on }\left\{x_{n} \leq 0\right\} \quad \text { and } \quad|D \phi| \leq \frac{c}{\delta} .
$$

Then we consider that

$$
\begin{cases}D_{i}\left(A_{i j}^{\epsilon}(x) D_{j} v_{\epsilon}\right)=0 & \text { in } B_{4}^{+}, \\ v_{\epsilon}=\phi w_{\epsilon} & \text { on } \partial B_{4}^{+} .\end{cases}
$$

According to [22], since $w_{\epsilon}=0$ on $\partial_{w} \Omega_{5}$,

$$
\int_{\Omega_{4} \cap\left\{x_{n} \leq 6 \delta\right\}}\left|\frac{w_{\epsilon}}{\delta}\right|^{2} d x \leq c \int_{\Omega_{4} \cap\left\{x_{n} \leq 6 \delta\right\}}\left|D w_{\epsilon}\right|^{2} d x
$$

Applying the $L^{2}$ estimate to (3.9) with (3.8) and (3.10), we have

$$
\frac{1}{\left|B_{4}\right|} \int_{B_{4}^{+}}\left|D v_{\epsilon}\right|^{2} d x \leq \frac{c}{\left|B_{4}\right|} \int_{B_{4}^{+}}\left|D\left(\phi w_{\epsilon}\right)\right|^{2} d x \leq \frac{c}{\left|B_{4}\right|} \int_{B_{4}^{+}}\left|D w_{\epsilon}\right|^{2} d x \leq c
$$

for some constant $c=c(\lambda, \Lambda, n)$. 
Since $v_{\epsilon}=0$ on $T_{4}$, we denote $\bar{v}_{\epsilon}$ to be the zero extension of $\tilde{v}_{\epsilon}$ from $B_{4}^{+}$to $B_{4}$. By the uniform ellipticity (1.2),

$$
\begin{aligned}
\frac{1}{\left|B_{4}\right|} \int_{B_{4}^{+}}\left|D\left(v_{\epsilon}-\phi w_{\epsilon}\right)\right|^{2} d x \leq & \frac{c}{\left|B_{4}\right|} \int_{\Omega_{4}} A_{i j}^{\epsilon} D_{i}\left(\bar{v}_{\epsilon}-\phi w_{\epsilon}\right) D_{j}\left(\bar{v}_{\epsilon}-\phi w_{\epsilon}\right) d x \\
= & \frac{c}{\left|B_{4}\right|} \int_{\Omega_{4}} A_{i j}^{\epsilon} D_{i}\left(\bar{v}_{\epsilon}-w_{\epsilon}\right) D_{j}\left(\bar{v}_{\epsilon}-\phi w_{\epsilon}\right) d x \\
& +\frac{c}{\left|B_{4}\right|} \int_{\Omega_{4}} A_{i j}^{\epsilon} D_{i}\left(w_{\epsilon}-\phi w_{\epsilon}\right) D_{j}\left(\bar{v}_{\epsilon}-\phi w_{\epsilon}\right) d x
\end{aligned}
$$

for some constant $c=c(\lambda)$. Since $w_{\epsilon}$ and $v_{\epsilon}$ are solutions to (3.5) and (3.9), respectively, by a direct computation, we see from (3.10) and (3.11) that

$$
\begin{aligned}
& \frac{1}{\left|B_{4}\right|} \int_{B_{4}^{+}}\left|D\left(v_{\epsilon}-\phi w_{\epsilon}\right)\right|^{2} d x \\
& \quad \leq \frac{c}{\left|B_{4}\right|} \int_{\Omega_{4}}\left|D\left(w_{\epsilon}-\phi w_{\epsilon}\right)\right|\left|D\left(\bar{v}_{\epsilon}-\phi w_{\epsilon}\right)\right| d x \\
& \quad=\frac{c}{\left|B_{4}\right|} \int_{\Omega_{4}}\left|D\left((1-\phi) w_{\epsilon}\right)\right|\left|D\left(\bar{v}_{\epsilon}-\phi w_{\epsilon}\right)\right| d x \\
& \quad \leq \frac{c}{\left|B_{4}\right|} \int_{\Omega_{4} \cap\left\{x_{n} \leq 6 \delta\right\}}\left(\left|D w_{\epsilon}\right|+\left|D\left(\phi w_{\epsilon}\right)\right|\right)\left|D\left(\bar{v}_{\epsilon}-\phi w_{\epsilon}\right)\right| d x \\
& \quad \leq \frac{c}{\left|B_{4}\right|}\left(\int_{\Omega_{4} \cap\left\{x_{n} \leq 6 \delta\right\}}\left|D w_{\epsilon}\right|^{2} d x\right)^{\frac{1}{2}}\left(\int_{B_{4}^{+}}\left|D\left(v_{\epsilon}-\phi w_{\epsilon}\right)\right|^{2} d x\right)^{\frac{1}{2}}
\end{aligned}
$$

for some constant $c=c(\lambda, \Lambda, n)$. Therefore,

$$
\frac{1}{\left|B_{4}\right|} \int_{B_{4}^{+}}\left|D\left(v_{\epsilon}-\phi w_{\epsilon}\right)\right|^{2} d x \leq \frac{c}{\left|B_{4}\right|} \int_{\Omega_{4} \cap\left\{x_{n} \leq 6 \delta\right\}}\left|D w_{\epsilon}\right|^{2} d x
$$

and hence

$$
\begin{aligned}
\frac{1}{\left|B_{4}\right|} \int_{B_{4}^{+}}\left|D\left(v_{\epsilon}-w_{\epsilon}\right)\right|^{2} d x & \leq \frac{c}{\left|B_{4}\right|} \int_{B_{4}^{+}}\left|D\left(v_{\epsilon}-\phi w_{\epsilon}\right)\right|^{2}+\left|D\left(\phi w_{\epsilon}-w_{\epsilon}\right)\right|^{2} d x \\
& \leq \frac{c}{\left|B_{4}\right|} \int_{\Omega_{4} \cap\left\{x_{n} \leq 6 \delta\right\}}\left|D w_{\epsilon}\right|^{2} d x .
\end{aligned}
$$

Finally, applying the higher integrability result in Lemma 3.2, Hölder's inequality and (3.8) to (3.12), we have

$$
\frac{1}{\left|B_{4}\right|} \int_{B_{4}^{+}}\left|D\left(v_{\epsilon}-w_{\epsilon}\right)\right|^{2} d x \leq c \delta^{\frac{\sigma_{1}}{2+\sigma_{1}}}
$$

for some constant $c=c(\lambda, \Lambda, n)$. Then we can choose a small constant $\delta$ satisfying $c \delta^{\frac{\sigma_{1}}{2+\sigma_{1}}}<\kappa^{2}$. This completes the proof.

Remark 3.5 We note that in the proof of Lemma 3.4, we did not use Lemma 3.3. In addition, we do not need any regularization on the coefficients in the proof of Lemma 3.4. In 
particular, the higher integrability result in Lemma 3.2 holds for the general cases not only for the homogenization problems. In this sense, we can see that Lemma 3.4 holds for the general cases that the coefficients only satisfy (1.2).

The next lemma is the main lemma in our argument. This verifies the second condition of Lemma 2.4 in the procedure to prove Theorem 3.1. In the following lemma, we let $c_{*}$ be the constant in Lemma 2.4 with respect to $w \in A_{s}$.

Lemma 3.6 Let $w \in A_{s}$ for some $1<s<\infty$, and let $u_{\epsilon}$ be the weak solution to (1.1). Then there exists a constant $\eta=\eta(\lambda, \Lambda, n, p, s, w)$ so that one can select small $\delta=\delta(\lambda, \Lambda, n, p, s, w)>$ 0 such that if $A$ is $(\delta, 96)$-vanishing, if $\Omega$ is $(\delta, 96)$-Reifenberg flat, and if for all $y \in \Omega$ and for every $0<\rho \leq 1, B_{\rho}(y)$ satisfies

$$
w\left(\left\{x \in \Omega: \mathcal{M}\left(\left|D u_{\epsilon}\right|^{2}\right)>N^{2}\right\} \cap B_{\rho}(y)\right)>\eta w\left(B_{\rho}(y)\right),
$$

where

$$
c_{*} N^{p} \eta=\frac{1}{2}
$$

then there holds

$$
\Omega \cap B_{\rho}(y) \subset\left\{x \in \Omega: \mathcal{M}\left(\left|D u_{\epsilon}\right|^{2}\right)>1\right\} \cup\left\{x \in \Omega: \mathcal{M}\left(|f|^{2}\right)>\delta^{2}\right\} .
$$

Proof We prove it by contradiction. Assume that $B_{\rho}(y)$ satisfies (3.13), but (3.15) is false. Then there is a point $y_{1} \in \Omega \cap B_{\rho}(y)$ such that

$$
\frac{1}{\left|B_{r}\left(y_{1}\right)\right|} \int_{\Omega_{r}\left(y_{1}\right)}\left|D u_{\epsilon}\right|^{2} d x \leq 1 \text { and } \frac{1}{\left|B_{r}\left(y_{1}\right)\right|} \int_{\Omega_{r}\left(y_{1}\right)}|f|^{2} d x \leq \delta^{2}
$$

for all $r>0$.

We split our problem into two cases: the interior case $B_{8 \rho}(y) \subset \Omega$ and the boundary case $B_{8 \rho}(y) \not \subset \Omega$. We here only consider the boundary case because the proof for the interior case is a simple modification of the proof for the boundary case.

We now assume $B_{8 \rho}(y) \not \subset \Omega$. Since our problem is invariant under rotation and translation, without loss of generality we can assume that

$$
B_{8 \rho}(y) \cap \Omega \subset B_{16 \rho} \cap \Omega
$$

and

$$
B_{96 \rho}^{+} \subset \Omega_{96 \rho} \subset B_{96 \rho} \cap\left\{x_{n}>-192 \delta\right\} .
$$

Then from (3.16) we can see that

$$
\frac{1}{\left|B_{96 \rho}\right|} \int_{\Omega_{96 \rho}}\left|D u_{\epsilon}\right|^{2} d x \leq \frac{\left|B_{192 \rho}\left(y_{1}\right)\right|}{\left|B_{96 \rho}\right|} \frac{1}{\left|B_{192 \rho}\right|} \int_{\Omega_{192 \rho}\left(y_{1}\right)}\left|D u_{\epsilon}\right|^{2} d x \leq 2^{n}
$$


and similarly

$$
\frac{1}{\left|B_{96 \rho}\right|} \int_{\Omega_{96 \rho}}|f|^{2} d x \leq 2^{n} \delta^{2}
$$

since $B_{96 \rho} \subset B_{192 \rho}\left(y_{1}\right)$.

Now we consider the following rescaled maps:

$$
\tilde{u}_{\epsilon}(z)=\frac{u_{\epsilon}(16 \rho z)}{16 \rho \sqrt{2^{n}}}, \quad \tilde{f}(z)=\frac{f(16 \rho z)}{16 \rho \sqrt{2^{n}}}, \quad \text { and } \quad \tilde{A}_{i j}^{\epsilon}(z)=A_{i j}^{\epsilon}(16 \rho z)
$$

for $z \in \tilde{\Omega}_{6}=\frac{1}{16 \rho} \Omega_{96 \rho}$. Then $\tilde{u}_{\epsilon}$ is a weak solution to

$$
\begin{cases}D_{i}\left(\tilde{A}_{i j}^{\epsilon}(z) D_{j} \tilde{u}_{\epsilon}(z)\right)=D_{\alpha} \tilde{f}_{i}^{\alpha}(z) & \text { in } \tilde{\Omega}_{6}, \\ \tilde{u}_{\epsilon}(z)=0 & \text { on } \partial_{w} \tilde{\Omega}_{6},\end{cases}
$$

satisfying

$$
\begin{aligned}
& B_{6}^{+} \subset \tilde{\Omega}_{6} \subset B_{6} \cap\left\{z_{n}>-12 \delta\right\}, \\
& \frac{1}{\left|B_{6}\right|} \int_{\tilde{\Omega}_{6}}\left|D \tilde{u}_{\epsilon}\right|^{2} d z \leq 1 \text { and } \frac{1}{\left|B_{6}\right|} \int_{\tilde{\Omega}_{6}}|\tilde{f}|^{2} d z \leq \delta^{2} .
\end{aligned}
$$

Next, we let $\tilde{w}_{\epsilon}$ be the weak solution to

$$
\begin{cases}D_{i}\left(\tilde{A}_{i j}^{\epsilon}(z) D_{j} \tilde{w}_{\epsilon}(z)\right)=0 & \text { in } \tilde{\Omega}_{5}, \\ \tilde{w}_{\epsilon}(z)=\tilde{u}_{\epsilon}(z) & \text { on } \partial \tilde{\Omega}_{5} .\end{cases}
$$

Comparing (3.19) with (3.18), the standard $L^{2}$ estimate yields

$$
\frac{1}{\left|B_{5}\right|} \int_{\tilde{\Omega}_{5}}\left|D \tilde{u}_{\epsilon}-D \tilde{w}_{\epsilon}\right|^{2} d z \leq \frac{c}{\left|B_{5}\right|} \int_{\tilde{\Omega}_{5}}|\tilde{f}|^{2} d z \leq c \delta^{2}
$$

for some constant $c=c(\lambda, \Lambda, n)$.

Now, we apply Lemma 3.4 to (3.19). Then, for any fixed $\kappa>0$, there exists small $\delta=$ $\delta(\kappa, \lambda, \Lambda, n)>0$ such that we can find a weak solution $\tilde{v}_{\epsilon}$ to

$$
\begin{cases}D_{i}\left(\tilde{A}_{i j}^{\epsilon}(z) D_{j} \tilde{v}_{\epsilon}(z)\right)=0 & \text { in } B_{4}^{+}, \\ \tilde{v}_{\epsilon}(z)=0 & \text { on } T_{4},\end{cases}
$$

satisfying

$$
\frac{1}{\left|B_{4}\right|} \int_{B_{4}^{+}}\left|D \tilde{v}_{\epsilon}\right|^{2} d z \leq c
$$

for some positive constant $c=c(\lambda, \Lambda, n)$ and

$$
\frac{1}{\left|B_{4}\right|} \int_{B_{4}^{+}}\left|D\left(\tilde{w}_{\epsilon}-\tilde{v}_{\epsilon}\right)\right|^{2} d z \leq \kappa^{2} .
$$


Note that according to the scaling in (3.17) $\tilde{A}$ is $(\delta, 6)$-vanishing, so we can apply Lemma 3.3 to (3.21) with $q=\frac{p+\tau}{\tau}$, where $\tau=\tau(n, s, w)$ is a constant in Lemma 2.6. Then there is a small $\delta=\delta(\lambda, \Lambda, n, p, s, w)$ such that

$$
\left(f_{B_{3}^{+}}\left|D \tilde{v}_{\epsilon}\right|^{\frac{p+\tau}{\tau}} d z\right)^{\frac{\tau}{p+\tau}} \leq c\left(f_{B_{4}^{+}}\left|D \tilde{v}_{\epsilon}\right|^{2} d z\right)^{\frac{1}{2}} \leq c
$$

for some positive constant $c=c(\lambda, \Lambda, n, p, s, w)$.

We now claim that if $N_{1}^{2} \geq 2^{n}$, then

$$
\left\{z \in \tilde{\Omega}_{1}: \mathcal{M}\left(\left|D \tilde{u}_{\epsilon}\right|^{2}\right)>N_{1}^{2}\right\} \subset\left\{z \in \tilde{\Omega}_{1}: \mathcal{M}_{B_{3}}\left(\left|D \tilde{u}_{\epsilon}\right|^{2}\right)>N_{1}^{2}\right\}
$$

To show this, we denote $z_{1}=\frac{y_{1}}{16 \rho}$ and let $z_{0}$ be a point in $\left\{z \in \tilde{\Omega}_{1}: \mathcal{M}_{B_{3}}\left(\left|D \tilde{u}_{\epsilon}\right|^{2}\right) \leq N_{1}^{2}\right\}$. If $r \leq 2$, we have $B_{r}\left(z_{0}\right) \subset B_{3}$ and

$$
\frac{1}{\left|B_{r}\right|} \int_{\tilde{\Omega}_{r}\left(z_{0}\right)}\left|D \tilde{u}_{\epsilon}\right|^{2} d z \leq \mathcal{M}_{B_{3}}\left(\left|D \tilde{u}_{\epsilon}\right|^{2}\right)\left(z_{0}\right) \leq N_{1}^{2}
$$

For $r>2$, as $z_{1} \in \tilde{\Omega}_{r}\left(z_{0}\right) \subset B_{2 r}\left(z_{1}\right)$, we obtain from (3.16)

$$
\frac{1}{\left|B_{r}\right|} \int_{\tilde{\Omega}_{r}(z)}\left|D \tilde{u}_{\epsilon}\right|^{2} d z \leq \frac{\left|B_{2 r}\right|}{\left|B_{r}\right|} \frac{1}{\left|B_{2 r}\right|} \int_{\tilde{\Omega}_{2 r}\left(z_{1}\right)}\left|D \tilde{u}_{\epsilon}\right|^{2} d z \leq 2^{n} \leq N_{1}^{2}
$$

Thus, $\mathcal{M}\left(\left|D \tilde{u}_{\epsilon}\right|^{2}\right)\left(z_{0}\right) \leq N_{1}^{2}$, and hence the claim is proved.

To complete our argument, we denote $\overline{\tilde{v}}_{\epsilon}$ to be the zero extension of $\tilde{v}_{\epsilon}$ from $B_{3}^{+}$to $B_{3}$, and let $N^{2}=N_{1}^{2} 2^{n}$ for large $N_{1} \geq 2^{n}$ to be determined later in the proof.

Using claim (3.25) and (3.17), we compute

$$
\begin{aligned}
& \frac{1}{\left|B_{\rho}\right|}\left|\left\{x \in \Omega: \mathcal{M}\left(\left|D u_{\epsilon}\right|^{2}\right)>N^{2}\right\} \cap B_{\rho}(y)\right| \\
& \leq \frac{16^{n}}{\left|B_{1}\right|}\left|\left\{z \in \tilde{\Omega}_{1}: \mathcal{M}\left(\left|D \tilde{u}_{\epsilon}\right|^{2}\right)>N_{1}^{2}\right\}\right| \\
& \leq \frac{16^{n}}{\left|B_{1}\right|}\left|\left\{z \in \tilde{\Omega}_{1}: \mathcal{M}_{B_{3}}\left(\left|D \tilde{u}_{\epsilon}\right|^{2}\right)>N_{1}^{2}\right\}\right| \\
& \leq \frac{16^{n}}{\left|B_{1}\right|}\left|\left\{z \in \tilde{\Omega}_{1}: \mathcal{M}_{B_{3}}\left(\left|D \tilde{u}_{\epsilon}-D \tilde{w}_{\epsilon}\right|^{2}\right)>\frac{N_{1}^{2}}{9}\right\}\right| \\
&+\frac{16^{n}}{\left|B_{1}\right|}\left|\left\{z \in \tilde{\Omega}_{1}: \mathcal{M}_{B_{3}}\left(\left|D \tilde{w}_{\epsilon}-D \overline{\tilde{v}}_{\epsilon}\right|^{2}\right)>\frac{N_{1}^{2}}{9}\right\}\right| \\
&+\frac{16^{n}}{\left|B_{1}\right|}\left|\left\{z \in \tilde{\Omega}_{1}: \mathcal{M}_{B_{3}}\left(\left|D \overline{\tilde{v}}_{\epsilon}\right|^{2}\right)>\frac{N_{1}^{2}}{9}\right\}\right| \\
& \leq \frac{c}{N^{2}} \int_{\tilde{\Omega}_{3}}\left|D \tilde{u}_{\epsilon}-D \tilde{w}_{\epsilon}\right|^{2} d z+\frac{c}{N^{2}} \int_{\tilde{\Omega}_{3}}\left|D \tilde{w}_{\epsilon}-D \overline{\tilde{v}}_{\epsilon}\right|^{2} d z+\frac{c}{N^{\frac{p+\tau}{\tau}}} \int_{B_{3}^{+}}\left|D \tilde{v}_{\epsilon}\right|^{\frac{p+\tau}{\tau}} d z \\
&= I_{1}+I_{2}+I_{3},
\end{aligned}
$$

where the constant $c=c(\lambda, \Lambda, n, p, s, w)$. 
From (3.20)

$$
I_{1} \leq \frac{c}{N^{2}} \delta^{2} .
$$

For $I_{2}$, we use Lemma 3.2 and (3.23) for given $\kappa>0$, then we obtain

$$
\begin{aligned}
I_{2} & \leq \frac{c}{N^{2}}\left(\frac{1}{\left|B_{1}\right|} \int_{B_{3}^{+}}\left|D \tilde{w}_{\epsilon}-D \tilde{v}_{\epsilon}\right|^{2} d z+\frac{1}{\left|B_{1}\right|} \int_{\tilde{\Omega}_{3} \backslash B_{3}^{+}}\left|D \tilde{w}_{\epsilon}\right|^{2} d z\right) \\
& \leq \frac{c}{N^{2}}\left(\kappa^{2}+\left(\int_{\tilde{\Omega}_{3}}\left|D \tilde{w}_{\epsilon}\right|^{2+\sigma_{1}} d z\right)^{\frac{2}{2+\sigma_{1}}}\left(\int_{\tilde{\Omega}_{3} \backslash B_{3}^{+}} d z\right)^{\frac{\sigma_{1}}{2+\sigma_{1}}}\right) \\
& \leq \frac{c}{N^{2}}\left(\kappa^{2}+\delta^{\frac{\sigma_{1}}{2+\sigma_{1}}}\right) .
\end{aligned}
$$

Finally, by (3.22) and (3.24),

$$
I_{3} \leq \frac{c}{N^{\frac{p+\tau}{\tau}}}\left(\int_{B_{3}^{+}}\left|D \tilde{v}_{\epsilon}\right|^{2} d z\right)^{\frac{p+\tau}{2 \tau}} \leq \frac{c}{N^{\frac{p+\tau}{\tau}}} .
$$

Therefore, we have from (3.14), (3.26), (3.27), and (3.28) that

$$
\begin{aligned}
I_{1}+I_{2}+I_{3} & \leq \frac{c}{N^{2}}\left(\kappa^{2}+\delta^{2}+\delta^{\frac{\sigma_{1}}{2+\sigma_{1}}}\right)+\frac{c}{N^{\frac{p+\tau}{\tau}}} \\
& =c \eta^{\frac{2}{p}}\left(\kappa^{2}+\delta^{2}+\delta^{\frac{\sigma_{1}}{2+\sigma_{1}}}\right)+c \eta^{\frac{p+\tau}{p \tau}} \\
& =\eta^{\frac{1}{\tau}}\left(c \eta^{\frac{2}{p}-\frac{1}{\tau}}\left(\kappa^{2}+\delta^{2}+\delta^{\frac{\sigma_{1}}{2+\sigma_{1}}}\right)+c \eta^{\frac{1}{p}}\right)
\end{aligned}
$$

for some constant $c=c(\lambda, \Lambda, n, p, s, w)$.

To derive a contradiction, we now use the relation between the weighted Lebesgue measure and the usual Lebesgue measure in (2.2) in the following way:

$$
\frac{w\left(\left\{x \in \Omega: \mathcal{M}\left(\left|D u_{\epsilon}\right|^{2}\right)>N^{2}\right\} \cap B_{\rho}(y)\right)}{w\left(B_{\rho}(y)\right)} \leq \alpha\left(\frac{\left|\left\{x \in \Omega: \mathcal{M}\left(\left|D u_{\epsilon}\right|^{2}\right)>N^{2}\right\} \cap B_{\rho}(y)\right|}{\left|B_{\rho}\right|}\right)^{\tau} .
$$

According to (3.29), we see that

$$
\begin{aligned}
& \frac{w\left(\left\{x \in \Omega: \mathcal{M}\left(\left|D u_{\epsilon}\right|^{2}\right)>N^{2}\right\} \cap B_{\rho}(y)\right)}{w\left(B_{\rho}(y)\right)} \\
& \leq \alpha\left(\eta^{\frac{1}{\tau}}\left(c \eta^{\frac{2}{p}-\frac{1}{\tau}}\left(\kappa^{2}+\delta^{2}+\delta^{\frac{\sigma_{1}}{2+\sigma_{1}}}\right)+c \eta^{\frac{1}{p}}\right)\right)^{\tau} \\
& \quad=\left(\alpha\left(c \eta^{\frac{2}{p}-\frac{1}{\tau}}\left(\kappa^{2}+\delta^{2}+\delta^{\frac{\sigma_{1}}{2+\sigma_{1}}}\right)+c \eta^{\frac{1}{p}}\right)^{\tau}\right) .
\end{aligned}
$$

We first take $\eta=\eta(\lambda, \Lambda, n, p, s, w)$ so that

$$
0<c \eta^{\frac{\tau}{p}} \leq \frac{1}{3^{\tau} \alpha} .
$$

Then we can select $N$ from (3.14). From this $\eta$, we next choose $\kappa=\kappa(\lambda, \Lambda, n, p, s, w)$ such that

$$
0<c \eta^{\frac{2 \tau}{p}-1} \kappa^{2 \tau} \leq \frac{1}{3^{\tau} \alpha} .
$$


Finally, we can find the corresponding $\delta=\delta(\lambda, \Lambda, n, p, s, w)$ for $\kappa$ selected as (3.31) that

$$
0<c \eta^{\frac{2 \tau}{p}-1}\left(\delta^{2}+\delta^{\frac{\sigma_{1}}{2+\sigma_{1}}}\right)^{\tau} \leq \frac{1}{3^{\tau} \alpha} .
$$

Consequently, from (3.14), (3.30), (3.31), and (3.32), we have, for such $N, \eta$, and $\delta$,

$$
w\left(\left\{x \in \Omega: \mathcal{M}\left(\left|D u_{\epsilon}\right|\right)^{2}>N^{2}\right\} \cap B_{\rho}(y)\right) \leq \eta w\left(B_{\rho}\right)
$$

which is a contradiction to (3.13). This completes the proof.

We are going to prove Theorem 3.1. For this, we now fix any $p \in(2, \infty)$ and let $\eta$ and $N$ be constants given by Lemma 3.6 with respect to $s=\frac{p}{2}$, and we denote by $c$ the constants that can be explicitly computed in terms of the known data such as $\Omega, \lambda, \Lambda, n, p$, and $w$.

Proof of Theorem 3.1 Firstly, we can assume that $\|f\|_{L_{w}^{p}(\Omega)} \leq \delta$ for $f \in L_{w}^{p}(\Omega)$ with $w \in A_{\frac{p}{2}}$ since our problem (1.1) is invariant under normalization. Then it suffices to show that

$$
\left\|D u_{\epsilon}\right\|_{L_{w}^{p}(\Omega)} \leq c
$$

Now, we note that

$$
\int_{\Omega}|f|^{2} d x \leq\left(\int_{\Omega}|f|^{p} w d x\right)^{\frac{2}{p}}\left(\int_{\Omega} w^{\frac{-2}{p-2}} d x\right)^{\frac{p-2}{p}} .
$$

Moreover, we let $d=\operatorname{diam}(\Omega)$, then $\Omega \subset B_{d}\left(x_{0}\right)$ for any $x_{0} \in \Omega$, and hence from the definition of the weight function $w \in A_{\frac{p}{2}}$ in (2.1)

$$
\begin{aligned}
\left(\int_{\Omega} w^{\frac{-2}{p-2}} d x\right)^{\frac{p-2}{p}} & \leq\left(\left(\int_{B_{d}\left(x_{0}\right)} w^{\frac{-2}{p-2}} d x\right)^{\frac{p}{2}-1}\right)^{\frac{2}{p}} \\
& \leq\left(\left(f_{B_{d}\left(x_{0}\right)} w d x\right)^{-1}[w]_{\frac{p}{2}}\left|B_{d}\right|^{\frac{p}{2}-1}\right)^{\frac{2}{p}} \\
& \leq\left(\frac{[w]_{\frac{p}{2}}\left|B_{d}\right|^{\frac{p}{2}}}{w\left(B_{d}\left(x_{0}\right)\right)}\right)^{\frac{2}{p}}
\end{aligned}
$$

From (3.33) and (3.34), we see from $w(\Omega) \leq w\left(B_{d}\left(x_{0}\right)\right)$ that

$$
\int_{\Omega}|f|^{2} d x \leq d^{n}\left|B_{1}\right|\left(\frac{[w] \frac{p}{2}}{w(\Omega)}\right)^{\frac{2}{p}}\|f\|_{L_{w}^{p}(\Omega)}^{2} \leq c \delta^{2} .
$$

In order to apply Lemma 2.3 and Lemma 2.4 , we denote

$$
\mathcal{C}=\left\{x \in \Omega: \mathcal{M}\left(\left|D u_{\epsilon}\right|^{2}\right)>N^{2}\right\}
$$

and

$$
\mathcal{D}=\left\{x \in \Omega: \mathcal{M}\left(\left|D u_{\epsilon}\right|^{2}\right)>1\right\} \cup\left\{x \in \Omega: \mathcal{M}\left(|f|^{2}\right)>\delta^{2}\right\} .
$$


Using (2.2), we know that, for any $y \in \Omega$,

$$
\frac{w\left(B_{1}(y) \cap \mathcal{C}\right)}{w\left(B_{1}(y)\right)} \leq \alpha\left(\frac{\left|B_{1}(y) \cap \mathcal{C}\right|}{\left|B_{1}(y)\right|}\right)^{\tau}
$$

Then by the weak $1-1$ estimate, the $L^{2}$-estimate and (3.35) yield

$$
\left|B_{1}(y) \cap \mathcal{C}\right| \leq \frac{c}{N^{2}} \int_{\Omega}\left|D u_{\epsilon}\right|^{2} d x \leq \frac{c}{N^{2}} \int_{\Omega}|f|^{2} d x \leq c \delta^{2}\left|B_{1}(y)\right|
$$

Then we can take small $\delta>0$ satisfying from (3.36) and (3.37) that

$$
w\left(B_{1}(y) \cap \mathcal{C}\right) \leq c \delta^{2 \tau}<\eta w\left(B_{1}(y)\right) .
$$

This shows that the first condition in Lemma 2.4 is satisfied with respect to $\eta$. Also, Lemma 3.6 verifies the other condition of Lemma 2.4. Therefore,

$$
w(\mathcal{C})<\eta_{1} w(\mathcal{D})
$$

where $\eta_{1}=c_{*} \eta$, and hence we obtain

$$
\begin{aligned}
& w\left(\left\{x \in \Omega: \mathcal{M}\left(\left|D u_{\epsilon}\right|^{2}\right)>N^{2}\right\}\right) \\
& \quad \leq \eta_{1} w\left(\left\{x \in \Omega: \mathcal{M}\left(\left|D u_{\epsilon}\right|^{2}\right)>1\right\}\right)+\eta_{1} w\left(\left\{x \in \Omega: \mathcal{M}\left(|f|^{2}\right)>\delta^{2}\right\}\right) .
\end{aligned}
$$

In addition, after iterating (3.38), we have

$$
\begin{aligned}
w(\{x \in & \left.\left.\Omega: \mathcal{M}\left(\left|D u_{\epsilon}\right|^{2}\right)>N^{2 m}\right\}\right) \\
\leq & \eta_{1}^{m} w\left(\left\{x \in \Omega: \mathcal{M}\left(\left|D u_{\epsilon}\right|^{2}\right)>1\right\}\right) \\
& +\sum_{i=1}^{m} \eta_{1}^{i} w\left(\left\{x \in \Omega: \mathcal{M}\left(|f|^{2}\right)>\delta^{2} N^{2(m-i)}\right\}\right) .
\end{aligned}
$$

Now we apply Lemma 2.3 to

$$
g=\mathcal{M}\left(\left|D u_{\epsilon}\right|^{2}\right), \quad \mu=N^{2}, \quad \theta=1, \quad \text { and } \quad q=\frac{p}{2} \text {. }
$$

By a direct computation we find that

$$
\begin{aligned}
& \left\|\mathcal{M}\left(\left|D u_{\epsilon}\right|^{2}\right)\right\|_{L_{w}^{\frac{p}{2}} \frac{p}{2}(\Omega)}^{\frac{p}{2}} \\
& \quad \leq c\left(w(\Omega)+\sum_{m \geq 1} N^{2 m \frac{p}{2}} w\left(\left\{x \in \Omega: \mathcal{M}\left(\left|D u_{\epsilon}\right|^{2}\right)>N^{2 m}\right\}\right)\right) \\
& \quad \leq c+c \sum_{m \geq 1} N^{m p} \eta_{1}^{m} w\left(\left\{x \in \Omega: \mathcal{M}\left(\left|D u_{\epsilon}\right|^{2}\right)>1\right\}\right)
\end{aligned}
$$




$$
\begin{aligned}
& +c \sum_{m \geq 1} N^{m p} \sum_{i=1}^{m} \eta_{1}^{i} w\left(\left\{x \in \Omega: \mathcal{M}\left(|f|^{2}\right)>\delta^{2} N^{2(m-i)}\right\}\right) \\
= & c+c w(\Omega) \sum_{m \geq 1} N^{m p} \eta_{1}^{m}+c \sum_{i \geq 1}\left(N^{p} \eta_{1}\right)^{i} \frac{\|f\|_{L_{w}^{p}(\Omega)}^{2}}{\delta^{2}} .
\end{aligned}
$$

Therefore

$$
\left\|\mathcal{M}\left(\left|D u_{\epsilon}\right|^{2}\right)\right\|_{L_{w}^{\frac{p}{2}}(\Omega)}^{\frac{p}{2}} \leq c\left(1+\sum_{m \geq 1}\left(N^{p} \eta_{1}\right)^{m}\right) \leq c
$$

since

$$
N^{p} \eta_{1}=c_{*} N^{p} \eta=\frac{1}{2}
$$

Then Lemma 2.7 shows that

$$
\left\|D u_{\epsilon}\right\|_{L_{w}^{p}(\Omega)} \leq c
$$

This completes the proof.

Remark 3.7 Our argument is based on the $L^{2}$ theory. In this sense, the condition $w \in A_{\frac{p}{2}}$ is natural, see (3.33). In fact, since

$$
\|f\|_{L_{w}^{p}(\Omega)}^{2}=\left(\int_{\Omega}|f|^{p} w d x\right)^{\frac{2}{p}}=\left(\int_{\Omega}\left(|f|^{\frac{p}{2}}\right)^{2} w d x\right)^{\frac{2}{p}}=\left\||f|^{2}\right\|_{L_{w}^{2}(\Omega)},
$$

it makes sense. Moreover, for the next two sections, we interpret Theorem 3.1 in the following way:

$$
w \in A_{p} \quad \text { and } \quad|f|^{2} \in L_{w}^{p}(\Omega) \quad \text { with } 1<p<\infty \quad \Rightarrow \quad\left|D u_{\epsilon}\right|^{2} \in L_{w}^{p}(\Omega)
$$

with the estimate

$$
\left\|\left|D u_{\epsilon}\right|^{2}\right\|_{L_{w}^{p}(\Omega)} \leq c\left\||f|^{2}\right\|_{L_{w}^{p}(\Omega)} .
$$

\section{Estimates in Orlicz spaces}

In this section, we are going to derive uniform Calderón-Zygmund estimates with respect to a given data $f$ from Orlicz spaces. Orlicz spaces are another natural generalization of the usual Lebesgue spaces, see [28, 29], but they are different from weighted Lebesgue spaces. For this reason, in order to focus on the estimates in Orlicz spaces, throughout this section, we let $w=1$ for $w d x$, that is, $w(E)=|E|$ for any measurable set $E \subset \mathbb{R}^{n}$, and we use Lemma 2.6 with $\alpha=1$ and $\tau=1$. In these settings, we apply lemmas in Sect. 3 to this section for our purpose. To describe Orlicz spaces, we start with some definitions.

Definition 4.1 Let $\Phi$ be a real-valued function defined on $[0, \infty)$. 
(1) We call $\Phi$ a Young function if it is a nondecreasing and convex function which satisfies

$$
\begin{aligned}
& \Phi(0)=0, \quad \lim _{\rho \rightarrow \infty} \Phi(\rho)=\infty, \\
& \lim _{\rho \rightarrow 0+} \frac{\Phi(\rho)}{\rho}=0, \quad \text { and } \quad \lim _{\rho \rightarrow \infty} \frac{\Phi(\rho)}{\rho}=\infty .
\end{aligned}
$$

(2) A Young function $\Phi$ satisfies $\Delta_{2}$-condition, denoted by $\Phi \in \Delta_{2}$, if there is a constant $c_{1}$ such that, for all $\rho \geq 0$,

$$
\Phi(2 \rho) \leq c_{1} \Phi(\rho)
$$

(3) A Young function $\Phi$ satisfies $\nabla_{2}$-condition, denoted by $\Phi \in \nabla_{2}$, if there is a constant $c_{2}$ such that, for all $\rho \geq 0$,

$$
\Phi(\rho) \leq \frac{\Phi\left(c_{2} \rho\right)}{2 c_{2}} .
$$

(4) Given a Young function $\Phi \in \Delta_{2} \cap \nabla_{2}$ and a bounded domain $U \in \mathbb{R}^{n}$, the Orlicz space $L^{\Phi}(U)$ is defined by the set of all measurable functions $g: U \rightarrow \mathbb{R}$ satisfying

$$
\int_{U} \Phi(|g|) d x<\infty
$$

equipped with the Luxemburg norm

$$
\|g\|_{L^{\Phi}(U)}=\inf \left\{\rho>0: \int_{U} \Phi\left(\frac{|g|}{\rho}\right) d x \leq 1\right\}
$$

Based on Definition 4.1, we can see that a Young function $\Phi \in \Delta_{2} \cap \nabla_{2}$ has growth conditions neither too slow nor too fast. In fact, $\Phi(\rho)=e^{\rho^{2}} \notin \Delta_{2}$ and $\Phi(\rho)=\rho \log (1+\rho) \notin$ $\nabla_{2}$. A typical example of $\Phi \in \Delta_{2} \cap \nabla_{2}$ is $\Phi(\rho)=\rho^{p}$ for $1<p<\infty$ that coincides with $L^{p}$ spaces, and hence the usual Lebesgue spaces are special cases of the Orlicz spaces. In addition, for $\Phi \in \Delta_{2} \cap \nabla_{2}$, there exist constants $q_{1}$ and $q_{2}$ with $1<q_{1} \leq q_{2}<\infty$ such that, for any $\rho_{1}$ and $\rho_{2}$ with $0<\rho_{1} \leq 1 \leq \rho_{2}<\infty$,

$$
\Phi\left(\rho_{1} \rho\right) \leq c \rho_{1}^{q_{1}} \Phi(\rho) \quad \text { and } \quad \Phi\left(\rho_{2} \rho\right) \leq c \rho_{2}^{q_{2}} \Phi(\rho) \quad \text { for all } \rho \geq 0
$$

for some constant $c$ independent of $\rho, \rho_{1}$, and $\rho_{2}$. In other words, for any $l>0$ and $\rho>0$,

$$
\frac{1}{c} \min \left\{l^{q_{1}}, l^{q_{2}}\right\} \leq \Phi(l \rho) \leq c \max \left\{l^{q_{1}}, l^{q_{2}}\right\}
$$

for some constant $c$ independent of $l$ and $\rho$. Then, from the definition of $\|g\|_{L^{\Phi}(\Omega)}$, for a bounded domain $\Omega$, we see that

$$
L^{q_{2}}(\Omega) \subset L^{\Phi}(\Omega) \subset L^{q_{1}}(\Omega) \subset L^{1}(\Omega)
$$


and $g \in L^{\Phi}(\Omega)$ implies $g \in L^{1}(\Omega)$ with the estimate

$$
\int_{\Omega}|g| d x \leq c\left(\left(\int_{\Omega} \Phi(|g|) d x\right)^{\frac{1}{q_{1}}}+\left(\int_{\Omega} \Phi(|g|) d x\right)^{\frac{1}{q_{2}}}\right),
$$

see $[3,30]$.

Similar to the weighted Lebesgue spaces, there is a fundamental property of the HardyLittlewood maximal function in the Orlicz spaces.

Lemma 4.2 ([23]) Let $\Phi$ be a Young function satisfying $\Phi \in \Delta_{2} \cap \nabla_{2}$ and $\Omega$ be a bounded domain. Then there is a constant $c=c(n, \Phi)>0$ such that

$$
c^{-1}\|g\|_{L^{\Phi}(\Omega)} \leq\|\mathcal{M} g\|_{L^{\Phi}(\Omega)} \leq c\|g\|_{L^{\Phi}(\Omega)}
$$

for all $g \in L^{\Phi}(\Omega)$.

Additionally, Lemma 2.2 for Orlicz spaces is the following.

Lemma 4.3 ([23]) Assume that $g$ is a nonnegative, measurable function defined on a bounded domain $\Omega \subset \mathbb{R}^{n}$. Let $\theta>0$ and $\mu>1$ be constants. Then, for any Young function $\Phi \in \Delta_{2} \cap \nabla_{2}$, we have

$$
g \in L^{\Phi}(\Omega) \quad \Longleftrightarrow \quad S=\sum_{k \geq 1} \Phi\left(\mu^{k}\right)\left|\left\{x \in \Omega: g(x)>\theta \mu^{k}\right\}\right|<\infty
$$

and

$$
\frac{1}{c} S \leq \int_{\Omega} \Phi(|g|) d x \leq c(|\Omega|+S)
$$

where the constant $c$ depends only on $\theta, \mu, q$, and $\Phi$.

The next is our theorem for the Orlicz spaces. In the proof of this, we mainly use properties (4.1) and (4.2) for the Orlicz space with respect to a Young $\Phi \in \Delta_{2} \cap \nabla_{2}$.

Theorem 4.4 Let $\Phi$ be a Young function satisfying $\Phi \in \Delta_{2} \cap \nabla_{2}$, and assume $|f|^{2} \in L^{\Phi}(\Omega)$. Then there exists a small positive constant $\delta=\delta(\lambda, \Lambda, n, p, \Phi)$ such that if $A=\left\{A_{i j}\right\}$ is $(\delta, 96)$ vanishing and $\Omega$ is $(\delta, 96)$-Reifenberg flat, then the weak solution $u_{\epsilon} \in H_{0}^{1}(\Omega)$ to (1.1) satisfies

$$
\left|D u_{\epsilon}\right|^{2} \in L^{\Phi}(\Omega)
$$

with the estimate

$$
\left\|\left|D u_{\epsilon}\right|^{2}\right\|_{L^{\Phi}(\Omega)} \leq c\left\||f|^{2}\right\|_{L^{\Phi}(\Omega)}
$$

for some constant $c=c(|\Omega|, \lambda, \Lambda, n, p, \Phi)$ which is independent of $\epsilon$. 
Proof In view of (4.2), according to the proofs in Lemma 3.6 with $w=1$ and $p=2 q_{2}$, we have the following: there exists a constant $\eta=\eta(\lambda, \Lambda, n, \Phi)$ so that one can select a small $\delta=\delta(\lambda, \Lambda, n, \Phi)>0$ such that if $A$ is $(\delta, 96)$-vanishing, if $\Omega$ is $(\delta, 96)$-Reifenberg flat, and if for all $y \in \Omega$ and for every $0<\rho \leq 1, B_{\rho}(y)$ satisfies

$$
\left|\left\{x \in \Omega: \mathcal{M}\left(\left|D u_{\epsilon}\right|^{2}\right)>N^{2}\right\} \cap B_{\rho}(y)\right|>\eta\left|B_{\rho}(y)\right|
$$

where

$$
c_{*} N^{2 q_{2}} \eta=\frac{1}{2}
$$

then there holds

$$
\Omega \cap B_{\rho}(y) \subset\left\{x \in \Omega: \mathcal{M}\left(\left|D u_{\epsilon}\right|^{2}\right)>1\right\} \cup\left\{x \in \Omega: \mathcal{M}\left(|f|^{2}\right)>\delta^{2}\right\} .
$$

Here, $c_{*}$ is the constant in Lemma 2.4 for $w=1$.

Similar to the proof of Theorem 3.1, we can assume that

$$
\left\||f|^{2}\right\|_{L^{\Phi}(\Omega)} \leq \delta^{2}
$$

for $|f|^{2} \in L^{\Phi}(\Omega)$, and hence it suffices to show that

$$
\left\|\left|D u_{\epsilon}\right|^{2}\right\|_{L^{\Phi}(\Omega)} \leq c
$$

As before, we let

$$
\mathcal{C}=\left\{x \in \Omega: \mathcal{M}\left(\left|D u_{\epsilon}\right|^{2}\right)>N^{2}\right\}
$$

and

$$
\mathcal{D}=\left\{x \in \Omega: \mathcal{M}\left(\left|D u_{\epsilon}\right|^{2}\right)>1\right\} \cup\left\{x \in \Omega: \mathcal{M}\left(|f|^{2}\right)>\delta^{2}\right\}
$$

Using (4.3) with the computation in (3.37), we can choose small $\delta$ satisfying for any $y \in \Omega$

$$
\left|B_{1}(y) \cap \mathcal{C}\right| \leq \frac{c}{N^{2}}\left(\delta^{\frac{1}{q_{1}}}+\delta^{\frac{1}{q_{2}}}\right)<\eta\left|B_{1}(y)\right|
$$

Then we can apply Lemma 2.4 for $w=1$ to our problem to see that

$$
\begin{aligned}
\mid\{x \in & \left.\Omega: \mathcal{M}\left(\left|D u_{\epsilon}\right|^{2}\right)>N^{2 m}\right\} \mid \\
\leq & \eta_{1}^{m}\left|\left\{x \in \Omega: \mathcal{M}\left(\left|D u_{\epsilon}\right|^{2}\right)>1\right\}\right| \\
& +\sum_{i=1}^{m} \eta_{1}^{i}\left|\left\{x \in \Omega: \mathcal{M}\left(|f|^{2}\right)>\delta^{2} N^{2(m-i)}\right\}\right|,
\end{aligned}
$$


where $\eta_{1}=c_{*} \eta$. Following from Lemma 4.3, we compute

$$
\begin{aligned}
& \int_{\Omega} \Phi\left(\mathcal{M}\left(\left|D u_{\epsilon}\right|^{2}\right)\right) d x \\
& \leq c\left(|\Omega|+\sum_{m \geq 1} \Phi\left(N^{2 m}\right)\left|\left\{x \in \Omega: \mathcal{M}\left(\left|D u_{\epsilon}\right|^{2}\right)>N^{2 m}\right\}\right|\right) \\
& \leq c+c \sum_{m \geq 1} \Phi\left(N^{2 m}\right) \eta_{1}^{m}\left|\left\{x \in \Omega: \mathcal{M}\left(\left|D u_{\epsilon}\right|^{2}\right)>1\right\}\right| \\
& \quad+c \sum_{m \geq 1} \Phi\left(N^{2 m}\right) \sum_{i=1}^{m} \eta_{1}^{i}\left|\left\{x \in \Omega: \mathcal{M}\left(|f|^{2}\right)>\delta^{2} N^{2(m-i)}\right\}\right| .
\end{aligned}
$$

Due to (4.1), we have $\Phi\left(N^{2 m}\right) \leq c N^{2 m q_{2}} \Phi(1)$, and then we obtain

$$
\int_{\Omega} \Phi\left(\mathcal{M}\left(\left|D u_{\epsilon}\right|^{2}\right)\right) d x \leq c+c|\Omega| \sum_{m \geq 1} N^{2 m q_{2}} \eta_{1}^{m}+c \sum_{i \geq 1}\left(N^{2 q_{2}} \eta_{1}\right)^{i}
$$

Since by (4.5)

$$
N^{2 q_{2}} \eta_{1}=c_{*} N^{2 q_{2}} \eta=\frac{1}{2}
$$

finally we have

$$
\int_{\Omega} \Phi\left(\mathcal{M}\left(\left|D u_{\epsilon}\right|^{2}\right)\right) d x \leq c
$$

for some constant $c=c(\lambda, \Lambda, n, \Phi, \Omega)$ independent of $\epsilon$. Then Lemma 4.2 shows that

$$
\left\|\left|D u_{\epsilon}\right|^{2}\right\|_{L^{\Phi}(\Omega)} \leq c .
$$

This completes the proof.

\section{Estimates in weighted Orlicz spaces}

In this section, we consider weighted Orlicz spaces. To do this, we let $\Phi$ be a Young function such that $\Phi \in \Delta_{2} \cap \nabla_{2}$. As in the previous section, for this $\Phi$ there exist constants $q_{1}$ and $q_{2}$ satisfying (4.1). We next define $i(\Phi)$ the lower index of a Young function $\Phi$ by

$$
i(\Phi)=\lim _{l \rightarrow 0+} \frac{\log \left(h_{\Phi}(l)\right)}{\log l}, \quad \text { where } h_{\Phi}(l)=\sup _{l>0} \frac{\Phi(l \rho)}{\Phi(\rho)} .
$$

Then we can see that the index number $i(\Phi)$ is equal to the supremum value of $q_{1}$ satisfying (4.1). We note that, as we have seen in Remark 3.7, in order to obtain uniform estimates in weighted Orlicz spaces, it is natural to consider the weight $w \in A_{i(\Phi)}$ for a Young function $\Phi \in \Delta_{2} \cap \nabla_{2}$ because of relation (4.2).

To apply our argument, we introduce the weighted Orlicz space $L_{w}^{\Phi}(\Omega)$ for the weight $w \in A_{i(\Phi)}$. The weighted Orlicz space $L_{w}^{\Phi}(\Omega)$ is defined by the set of all measurable functions $g: \Omega \rightarrow \mathbb{R}$ satisfying

$$
\int_{\Omega} \Phi(|g|) w d x<\infty
$$


equipped with the Luxemburg norm

$$
\|g\|_{L_{w}^{\Phi}(U)}=\inf \left\{\rho>0: \int_{U} \Phi\left(\frac{|g|}{\rho}\right) w d x \leq 1\right\} .
$$

Similar to (4.3) we see that $g \in L_{w}^{\Phi}(\Omega)$ implies $g \in L^{1}(\Omega)$ with the estimate

$$
\int_{\Omega}|g| w d x \leq c\left(\left(\int_{\Omega} \Phi(|g|) w d x\right)^{\frac{1}{q_{1}}}+\left(\int_{\Omega} \Phi(|g|) w d x\right)^{\frac{1}{q_{2}}}\right),
$$

see [30]. In these spaces, there is a fundamental property of the Hardy-Littlewood maximal function like Lemma 2.7 and Lemma 4.2, that is, there is a constant $c=c(n, w, \Phi)$ such that

$$
c^{-1}\|g\|_{L_{w}^{\Phi}(\Omega)} \leq\|\mathcal{M} g\|_{L_{w}^{\Phi}(\Omega)} \leq c\|g\|_{L_{w}^{\Phi}(\Omega)}
$$

for all $g \in L^{\Phi}(\Omega)$, see $[17,23]$. In addition, we have the following relation between integration and summation in $L_{w}^{\Phi}(\Omega)$.

Lemma 5.1 Assume that $g$ is a nonnegative, measurable function defined on a bounded domain $\Omega \subset \mathbb{R}^{n}$. Let $\theta>0$ and $\mu>1$ be constants. Then, for any Young function $\Phi \in \Delta_{2} \cap \nabla_{2}$ and corresponding $w \in A_{i(\Phi)}$, we have

$$
g \in L_{w}^{\Phi}(\Omega) \quad \Longleftrightarrow \quad S=\sum_{k \geq 1} \Phi\left(\mu^{k}\right) w\left(\left\{x \in \Omega: g(x)>\theta \mu^{k}\right\}\right)<\infty
$$

and

$$
\frac{1}{c} S \leq \int_{\Omega} \Phi(|g|) w d x \leq c(w(\Omega)+S),
$$

where the constant $c$ depends only on $\theta, \mu, q, w$, and $\Phi$.

The main result in this section is the following.

Theorem 5.2 Let $\Phi$ be a Young function satisfying $\Phi \in \Delta_{2} \cap \nabla_{2}$, and let $w \in A_{i(\Phi)}$. Assume $|f|^{2} \in L_{w}^{\Phi}(\Omega)$. Then there exists a small positive constant $\delta=\delta(\lambda, \Lambda, n, p, w, \Phi)$ such that if $A=\left\{A_{i j}\right\}$ is $(\delta, 96)$-vanishing and $\Omega$ is $(\delta, 96)$-Reifenberg flat, then the weak solution $u_{\epsilon} \in$ $H_{0}^{1}(\Omega)$ to $(1.1)$ satisfies

$$
\left|D u_{\epsilon}\right|^{2} \in L_{w}^{\Phi}(\Omega)
$$

with the estimate

$$
\left\|\left|D u_{\epsilon}\right|^{2}\right\|_{L_{w}^{\Phi}(\Omega)} \leq c\left\||f|^{2}\right\|_{L_{w}^{\Phi}(\Omega)}
$$

for some constant $c=c(\operatorname{diam}(\Omega), \lambda, \Lambda, n, p, w, w(\Omega), \Phi)$, which is independent of $\epsilon$. 
Proof The proof of this theorem is a combined one of the proofs in Theorem 3.1 and Theorem 4.4, so here we explain briefly the procedure of the proof.

After a suitable normalization, as the proof of Theorem 4.4, we can prove Lemma 3.6 for $w \in A_{i(\Phi)}$ and $p=q_{2}$, where $q_{2}$ is the constant in (4.1), in the same way. Then we can apply Lemma 2.4 for $w \in A_{i(\Phi)}$ to our problem, and hence we finally obtain by using Lemma 5.1 that

$$
\int_{\Omega} \Phi\left(\mathcal{M}\left(\left|D u_{\epsilon}\right|^{2}\right)\right) w d x \leq c+c \sum_{i \geq 1}\left(N^{2 q_{2}} \eta_{1}\right)^{i}
$$

for some constant $c=c(\lambda, \Lambda, n, w, \Phi)$ independent of $\epsilon$, where

$$
N^{2 q_{2}} \eta_{1}=c_{*} N^{2 q_{2}} \eta=\frac{1}{2}
$$

This completes the proof.

\section{Acknowledgements}

The author is very grateful to the handling editors and the anonymous reviewers for the careful reading of this manuscript and offering valuable comments for this manuscript.

\section{Funding}

This work was supported by 2020 Research Grant from Kangwon National University and the National Research

Foundation of Korea(NRF) grant funded by the Korea government(MSIT) (No. NRF-2020R1F1A1A01050243).

\section{Availability of data and materials}

Not applicable.

\section{Competing interests}

The author declares that they have no competing interests.

\section{Authors' contributions}

The author conceived of the study, drafted the manuscript, and approved the final manuscript.

\section{Publisher's Note}

Springer Nature remains neutral with regard to jurisdictional claims in published maps and institutional affiliations.

Received: 6 December 2020 Accepted: 25 February 2021 Published online: 10 March 2021

\section{References}

1. Avellaneda, M., Lin, F.: Compactness methods in the theory of homogenization. Commun. Pure Appl. Math. 40(6), 803-847 (1987)

2. Bensoussan, A., Lions, J.L., Papanicolaou, G.: Asymptotic Analysis for Periodic Structures, p. xii+398 AMS, Providence (2011). ISBN 978-0-8218-5324-5

3. Byun, S.-S., Cho, Y.: Nonlinear gradient estimates for parabolic obstacle problems in non-smooth domains. Manuscr. Math. 146(3-4), 539-558 (2015)

4. Byun, S.-S., Jang, Y.: Global W', estimates for elliptic systems in homogenization problems in Reifenberg domains. Ann. Mat. Pura Appl. (4) 195(6), 2061-2075 (2016)

5. Byun, S.-S., Jang, Y:: Calderón-Zygmund estimate for homogenization of parabolic systems. Discrete Contin. Dyn. Syst. 36(12), 6689-6714 (2016)

6. Byun, S.-S., Jang, Y.: Homogenization of the conormal derivative problem for elliptic systems in Reifenberg domains. Commun. Contemp. Math. 20(2), 1650062 (2018)

7. Byun, S.-S., Lee, M.: Weighted estimates for nondivergence parabolic equations in Orlicz spaces. J. Funct. Anal. 269(8), 2530-2563 (2015)

8. Byun, S.-S., Lee, M., Palagachev, D.K.: Hessian estimates in weighted Lebesgue spaces for fully nonlinear elliptic equations. J. Differ. Equ. 260(5), 4550-4571 (2016)

9. Byun, S.-S., Palagachev, D.K.: Weighted $L^{p}$-estimates for elliptic equations with measurable coefficients in nonsmooth domains. Potential Anal. 41(1), 51-79 (2014)

10. Byun, S.-S., Ryu, S.: Global estimates in Orlicz spaces for the gradient of solutions to parabolic systems. Proc. Am. Math Soc. 138(2), 641-653 (2010)

11. Byun, S.-S., Ryu, S.: Gradient estimates for higher order elliptic equations on nonsmooth domains. J. Differ. Equ. 250(1), 243-263 (2011) 
12. Byun, S.-S., So, H.: Weighted estimates for generalized steady Stokes systems in nonsmooth domains. J. Math. Phys. 58(2), $023101(2017)$

13. Caffarelli, L.A., Cabré, X.: Fully Nonlinear Elliptic Equations. American Mathematical Society Colloquium Publications, vol. 43. Am. Math. Soc., Providence (1995)

14. Caffarelli, L.A., Peral, I.: On W $W^{1, p}$ estimates for elliptic equations in divergence form. Commun. Pure Appl. Math. 51(1), $1-21(1998)$

15. Dong, H., Kim, D.: Elliptic and parabolic equations with measurable coefficients in weighted Sobolev spaces. Adv. Math. 274, 681-735 (2015)

16. Dong, H., Kim, D.: On $L_{p}$-estimates for elliptic and parabolic equations with $A_{p}$ weights. Trans. Am. Math. Soc. 370(7), 5081-5130 (2018)

17. Fiorenza, A., Krbec, M.: Indices of Orlicz spaces and some applications. Comment. Math. Univ. Carol. 38(3), 433-451 (1997)

18. Geng, J., Shen, Z:: Uniform regularity estimates in parabolic homogenization. Indiana Univ. Math. J. 64(3), 697-733 (2015)

19. Jia, H., Li, D., Wang, L.: Regularity theory in Orlicz spaces for elliptic equations in Reifenberg domains. J. Math. Anal. Appl. 334, 804-817 (2007)

20. Jia, H., Wang, L., Yao, F., Zhou, S.: Optimal regularity for the Poisson equation. Proc. Am. Math. Soc. 137(6), 2037-2047 (2009)

21. Kilpeläinen, T., Koskela, P.: Global integrability of the gradients of solutions to partial differential equations. Nonlinear Anal. 23(7), 899-909 (1994)

22. Kim, Y., Ryu, S.: Global gradient estimates for parabolic equations with measurable nonlinearities. Nonlinear Anal. 164 77-99 (2017)

23. Kokilashvili, V., Krbec, M.: Weighted Inequalities in Lorentz and Orlicz Spaces, p. xii+233 World Scientific, River Edge (1991). ISBN 981-02-0612-7

24. Maz'ya, V.G.: Sobolev Spaces. With Applications to Elliptic Partial Differential Equations, Grundlehren der Mathematischen Wissenschaften, vol. 342 (2nd revised and augmented ed.), p. xxviii+866. Springer, Berlin (2011). ISBN 978-3-642-15563-5

25. Mengesha, T., Phuc, N.C.: Weighted and regularity estimates for nonlinear equations on Reifenberg flat domains. J. Differ. Equ. 250(5), 2485-2507 (2011)

26. Muckenhoupt, B.: Weighted norm inequalities for the Hardy maximal function. Trans. Am. Math. Soc. 165, 207-226 (1972)

27. Phuc, N.C.: Nonlinear Muckenhoupt-Wheeden type bounds on Reifenberg flat domains, with applications to quasilinear Riccati type equations. Adv. Math. 250, 387-419 (2014)

28. Rao, M.M., Ren, Z.D.: Theory of Orlicz Spaces. Monographs and Textbooks in Pure and Applied Mathematics, vol. 146, p. xii+449 Dekker, New York (1991). ISBN 0-8247-8478-2

29. Rao, M.M., Ren, Z.D.: Applications of Orlicz Spaces. Monographs and Textbooks in Pure and Applied Mathematics, vol. 250, p. xii+464 Dekker, New York (2002). ISBN 0-8247-0730-3

30. Ryu, S.: Global gradient estimates for nonlinear elliptic equations. J. Korean Math. Soc. 51(6), 1209-1220 (2014)

31. Shen, Z:: $W^{1, p}$ estimates for elliptic homogenization problems in nonsmooth domains. Indiana Univ. Math. J. 57(5), 2283-2298 (2008)

32. Sun, Y., Yao, F., Zhou, S.: Gradient estimates in Orlicz spaces for quasilinear elliptic equation. Nonlinear Anal. 69(8), $2553-2565(2008)$

33. Toro, T:: Doubling and flatness: geometry of measures. Not. Am. Math. Soc. 44(9), 1087-1094 (1997)

34. Wang, L., Yao, F.: Higher-order nondivergence elliptic and parabolic equations in Sobolev spaces and Orlicz spaces. J. Funct. Anal. 262(8), 3495-3517 (2012)

35. Yao, F.: Global weighted $W^{2, p}$ estimates for nondivergence elliptic equations with small BMO coefficients. Int. J. Math. 26(11), 1550089 (2015)

\section{Submit your manuscript to a SpringerOpen ${ }^{\circ}$ journal and benefit from:}

- Convenient online submission

- Rigorous peer review

- Open access: articles freely available online

- High visibility within the field

- Retaining the copyright to your article

Submit your next manuscript at $\gg$ springeropen.com 